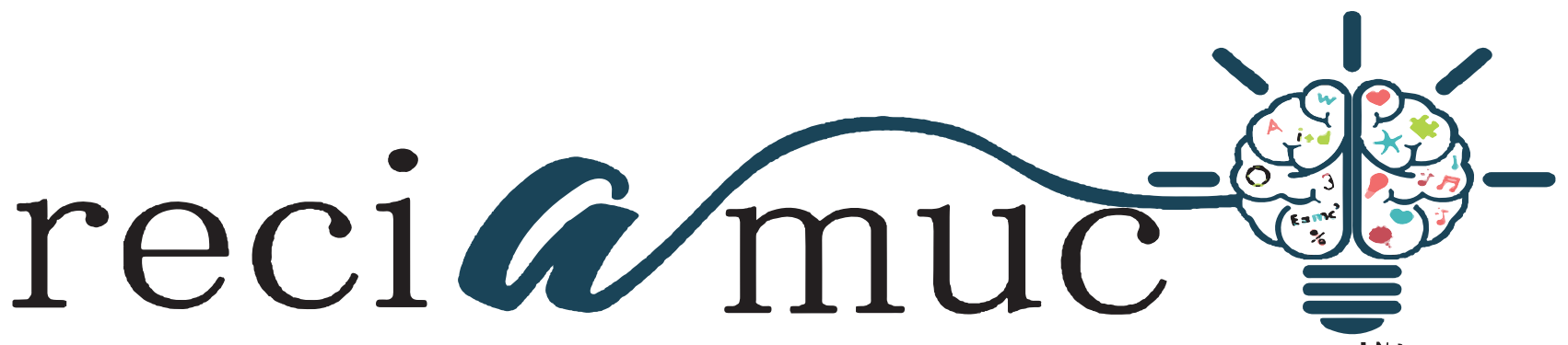

DOI: $10.26820 /$ reciamuc/4.(3).julio.2020.268-274

URL: https://reciamuc.com/index.php/RECIAMUC/article/view/529

EDITORIAL: Saberes del Conocimiento

REVISTA: RECIAMUC

ISSN: 2588-0748

TIPO DE INVESTIGACIÓN: Artículo de Revisión

Código UNESCO: 32 Ciencias Médicas

PAGINAS: 268-274

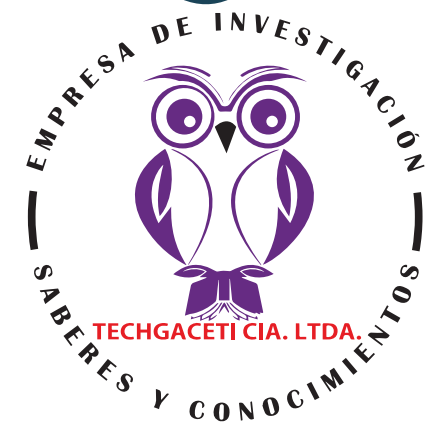

\title{
Tamizaje neonatal de hipotiroidismo congénito
}

Neonatal screening for congenital hypothyroidism

Alternativas de cuidados para evitar problemas de câncer de pele

\section{Dennis Gonzalo Calle Rueda'; Teresa Victoria Muñoz Cueva²; Carla Rocío Delgado Núñez; Rodrigo Sebastián Vera Enríquez ${ }^{4}$}

RECIBIDO: 20/05/2020 ACEPTADO: 19/07/2020 PUBLICADO: 30/07/2020

1. Médico; Investigador Independiente; Quito, Ecuador; dennis.calle2803@gmail.com; (iD https://orcid.org/0000-0002-52783444

2. Médico; Investigador Independiente; Quito, Ecuador; terevico25@gmail.com; (iD https://orcid.org/0000-0001-8297-1799

3. Médico; Investigador Independiente; Quito, Ecuador; carladelgado455@gmail.com; (D) https://orcid.org/0000-0003-4173306X

4. Médico; Investigador Independiente; Quito, Ecuador; renriquez@yahoo.es; (ID https://orcid.org/0000-0002-9193-097X

\section{CORRESPONDENCIA}

Dennis Gonzalo Calle Rueda

dennis.calle2803@gmail.com

Quito, Ecuador

() RECIAMUC; Editorial Saberes del Conocimiento, 2020 


\section{RESUMEN}

La posibilidad de medir las hormonas tiroideas a partir de sangre secada en papel de filtro abrió el camino para identificar a los recién nacidos con hipotiroidismo congénito $(\mathrm{HC})$ en los primeros días de vida. En consecuencia, a través de una prueba de cribado temprana, se puede dar inicio a una terapia de reemplazo adecuada, que ayuda a prevenir de manera eficaz el retraso mental. Este tipo de pruebas es muy sencilla, y consiste en hacer una punción en el talón del bebé con el fin de tomar la muestra de sangre y colocarla en el papel especial para su posterior análisis en el laboratorio. La prueba debe hacerse dentro de los primeros 30 días de nacido para determinar algún desorden metabólico que no es perceptible al momento del nacimiento y que puede ser tratado de inmediato. La recogida oportuna y completa de muestras, la logística de aporte, el análisis rápido y la comunicación de los resultados, son puntos clave para la organización de un programa de cribado neonatal de HC. Es necesaria una estrecha colaboración entre el laboratorio y los especialistas en tratamiento para garantizar un tratamiento y seguimiento adecuado de los bebés identificados por los programas de detección de HC.

Palabras clave: Hipotiroidismo congénito, Neonato, Hormonas tiroideas, Tamizaje.

\section{ABSTRACT}

The possibility of measuring thyroid hormones from blood dried on filter paper opened the way to identify newborprueba de cribado with congenital hypothyroidism $(\mathrm{CH})$ in the first days of life. Coprueba de cribadoequently, early initiation of appropriate replacement therapy helps to effectively prevent mental retardation. This type of test is very simple, and coprueba de cribadoists of making a puncture in the baby's heel in order to take the blood sample and place it on the special paper for later analysis in the laboratory. The test must be done within the first 30 days of birth to determine any metabolic disorder that is not noticeable at birth and that can be treated immediately. Timely and complete sample collection, traprueba de cribadoportation logistics, rapid analysis, and communication of results are key points for organizing a neonatal screening program for $\mathrm{CH}$. Close collaboration between the laboratory and treatment specialists is necessary to eprueba de cribadoure adequate treatment and follow-up of the babies identified by the HC screening programs.

Keywords: Congenital hypothyroidism, Neonate, Thyroid hormones, Screening.

\section{RESUMO}

A possibilidade de medir os hormônios tireoidianos a partir do sangue seco em papel filtro abriu caminho para a identificação de newborprueba de cribado com hipotireoidismo congênito (HC) nos primeiros dias de vida. Da mesma forma, o início precoce da terapia de reposição adequada ajuda a prevenir o retardo mental com eficácia. Esse tipo de exame é muito simples, sendo coprueba de cribadoístas de fazer uma punção no calcanhar do bebê a fim de colher a amostra de sangue e colocá-la no papel especial para posterior análise em laboratório. O teste deve ser feito nos primeiros 30 dias após o nascimento para determinar qualquer distúrbio metabólico que não seja perceptível ao nascimento e que possa ser tratado imediatamente. Coleta de amostra completa e oportuna, logística de traprueba de cribadoportation, análise rápida e comunicação dos resultados são pontos-chave para organizar um programa de triagem neonatal para HC. É necessária uma estreita colaboração entre o laboratório e os especialistas em tratamento para eprueba de cribadoure tratamento adequado e acompanhamento dos bebês identificados pelos programas de triagem do $\mathrm{HC}$.

Palavras-chave: Hipotireoidismo congênito, Neonatos, Hormônios tireoidianos, Rastreio. 


\section{Introducción}

El hipotiroidismo congénito $(\mathrm{HC})$ es una de las causas prevenibles más frecuentes de retraso mental. Por lo tanto, era solo cuestión de tiempo hasta que se desarrollara e introdujera una prueba de cribado neonatal adecuada para el diagnóstico temprano de HC. Los autores Dussault, Coulombe, \& Laberge, (1975) exponen que "el primer programa de cribado de HC se realizo en 1974 en Quebec (Canadá)", en tal sentido en el 2014 se cumplen 40 años de la aplicación de la prueba de cribado para HC estableciéndose a nivel mundial tanto en países desarrollados como en países en desarrollo.

La detección de HC es considerada uno de los pocos programas de medicina preventiva que tiene un impacto en la salud pública con una relación costo-beneficio positiva. "A principios de la década de 1970, se publicaron informes que describían la eficacia de la intervención temprana en el tratamiento del HC" (Rodríguez, María, \& Guindulain, 2019). Casi simultáneamente aparecieron informes que describían la posibilidad de medir las hormonas tiroideas a partir de la sangre del cordón umbilical o de manchas de sangre (DBS).

La estimulación cerebral profunda ya se estaba utilizando para recolectar muestras de prueba de cribado para fenilcetonuria y se convirtió inmediatamente en el material de muestra de elección para el cribado de HC. "Si bien la elección del dispositivo de muestreo (la tarjeta de papel de filtro para DBS) fue ampliamente aceptada, la elección de qué hormona tiroidea debe medirse, es decir, hormona estimulante de la tiroides (TSH) o tiroxina (T 4), fue objeto de unos argumentos". (Marrero \& Frometa, 2000)

La controversia en ese momento se centró principalmente en la especificidad en comparación con la prueba de cribado. En tal sentido, Marrero \& Frometa, (2000) exponen que la "TSH era el analito óptimo para detectar el hipotiroidismo primario, pero habían limitaciones en las pruebas de cribado y los de TSH, debido a una prueba de cribado relativamente pobre". Por el contrario, las pruebas de cribado de T4 fueron algo más certeras, sin embargo, se dice que también carecían de especificidad y dieron lugar a frecuentes resultados falsos positivos, especialmente en bebés prematuros.

Al inicio, "el uso de T4 como marcador de detección era común en América del Norte, mientras que la TSH era el analito de elección en Europa y Japón" (Medline Plus, 2020). Hoy en día, el cribado de HC está en funcionamiento en la mayor parte del mundo y es, junto con el cribado de fenilcetonuria, el primer programa que se implementa en los países que recién comienzan un programa de prueba de cribado neonatal (PCN) o tamizaje neonatal.

\section{Metodología}

Para el desarrollo de este proceso investigativo, se plantea como metodología la encaminada hacia una orientación científica particular que se encuentra determinada por la necesidad de indagar en forma precisa y coherente una situación, en tal sentido Davila, (2015) define la metodología "como aquellos pasos anteriores que son seleccionados por el investigador para lograr resultados favorables que le ayuden a plantear nuevas ideas" (p.66)

Lo citado por el autor, lleva a entender que el desarrollo de la acción investigativa busca simplemente coordinar acciones enmarcadas en una revisión bibliográfica con el fin de complementar ideas previas relacionadas Tamizaje neonatal de hipotiroidismo congénito a través de una revisión de literatura, para así finalmente elaborar un cuerpo de consideraciones generales que ayuden a ampliar el interés propuesto.

\section{Tipo de Investigación}

Dentro de toda práctica investigativa, se precisan acciones de carácter metodológico mediante las cuales se logra conocer y proyectar los eventos posibles que la determinan. En este sentido, la presente investigación corresponde al tipo documental, definido por Castro (2016), "se ocupa del estudio de problemas planteados a nivel teórico, la información requerida para abordarlos se encuentra básicamente en materiales impresos, audiovisuales y / o electrónicos". (p.41).

En consideración a esta definición, la orientación metodológica incluye la oportunidad de cumplir con una serie de actividades inherentes a la revisión y lectura de diversos documentos, donde se encuentran ideas explicitas relacionadas con los tópicos encargados de identificar una característica inmersa en el estudio. Por lo tanto, se realizaron continuas interpretaciones con el claro propósito de revisar aquellas apreciaciones propuestas por diferentes investigadores en relación al tema de interés, para luego dar la respectiva argumentación a los planteamientos, en función a las necesidades encontradas en la investigación, apoyados en las herramientas tecnológicas para la búsqueda de trabajos con valor científico disponibles en la web que tenían conexión con el objetivo principal de la investigación.

\section{Fuentes Documentales}

El análisis correspondiente a las características que predomina en el tema seleccionado, llevan a incluir diferentes fuentes documentales encargadas de darle el respectivo valor científico y en ese sentido cumplir con la valoración de los hechos a fin de generar nuevos criterios que sirven de referencia a otros procesos investigativos. Para Castro,(2016) las 
fuentes documentales incorporadas en la investigación documental o bibliográfica, "representa la suma de materiales sistemáticos que son revisados en forma rigurosa y profunda para llegar a un análisis del fenómeno" (p.41). Por lo tanto, se procedió a cumplir con la lectura previa determinada para encontrar aquellos aspectos estrechamente vinculados con el tema, con el fin de explicar mediante un desarrollo las respectivas apreciaciones generales de importancia.

\section{Técnicas para la Recolección de la Información}

La conducción de la investigación para ser realizada en función a las particularidades que determinan a los estudios documentales, tiene como fin el desarrollo de un conjunto de acciones encargadas de llevar a la selección de técnicas estrechamente vinculadas con las características del estudio. Bolívar, (2015), refiere, que es "una técnica particular para aportar ayuda a los procedimientos de selección de las ideas primarias y secundarias". (p.71).

Tal como lo expresa, Bolívar, (2015) "Las técnicas documentales proporcionan las herramientas esenciales y determinantes para responder a los objetivos formulados y llegar a resultados efectivos" (p. 58). Es decir, para responder con eficiencia a las necesidades investigativas, se introdujeron como técnica de recolección el método inductivo, que hizo posible llevar a cabo una valoración de los hechos de forma particular para llegar a la explicación desde una visión general. El autor Bolívar, (2015) tambien expresa que las técnicas de procesamiento de datos en los estudios documentales "son las encargadas de ofrecer al investigador la visión o pasos que deben cumplir durante su ejercicio, cada una de ellas debe estar en correspondencia con el nivel a emplear" ( $p$. 123). Esto indica, que para llevar a cabo el procesamiento de los datos obtenidos una vez aplicadas las técnicas seleccionadas, tales como: fichas de resumen, textual, registros descriptivos entre otros, los mismos se deben ajustar al nivel que ha sido seleccionado.

\section{Resultados}

\section{Organización de cribado}

Las pruebas de cribado en general, son una iniciativa de salud pública. Por lo tanto, la mayoría de los programas están organizados y financiados por los respectivos departamentos de salud. En algunas jurisdicciones, "la participación en la selección es obligatoria y está regulada por la ley, en otras se basa en la prueba de cribado informada, esto significa que los padres tienen la posibilidad de optar por no participar en prueba de cribado" (Rodríguez, María, \& Guindulain, 2019). El cribado es único en el sentido de que no se realiza como parte de la investigación o el tratamiento de personas sintomáticas, pero generalmente se ofrece a todas las personas de una comunidad que no se sabe que corren el riesgo de padecer la afección. Esto implica que el PCN no es una prueba diagnóstica y los resultados de la misma no deben tratarse como tal y, por lo tanto, confirmarse antes de la intervención.

La PCN generalmente se realiza en grandes unidades de laboratorio, procesando varios miles de muestras por año. Idealmente, dicho laboratorio debería estar ubicado en una instalación académica, preferiblemente pediátrica. "Se ha establecido un tamaño mínimo para un laboratorio de prueba de cribado en el rango de alrededor de 50.000 a 100.000 muestras por año" (Rodríguez, Sanz, \& Dulín, 2017). El fundamento de tal recomendación se deriva del hecho de que con menos de 50.000 muestras, el proceso de selección puede volverse ineficaz.

Un número reducido de muestras puede dar lugar a una relación costo-beneficio deficiente y proporcionar una base insuficiente para el análisis estadístico. Un laboratorio de prueba de cribado también debe participar en programas de control de calidad externos, idealmente en uno internacional como el Programa de Garantía de Calidad de Detección de Recién Nacidos dirigido por los Centros para el Control de Enfermedades.

Para que un laboratorio de PCN funcione adecuadamente, una comunicación continua con los especialistas involucrados en el cuidado de los recién nacidos con resultados positivos de detección es de suma importancia y también es parte del proceso de control de calidad. En tal sentido, Rodríguez, Sanz, \& Dulín, (2017) afirman que "cuanto más cortas sean las líneas de comunicación entre el laboratorio y el especialista tratante, mejor será el resultado del proceso de detección". Adicionalmente, los resultados patológicos del cribado y el resultado del caso correspondiente deberían sentarse en un registro nacional centralizado, a fin de permitir los estudios epidemiológicos.

\section{Estrategia de cribado}

La detección de HC primario se ha introducido en la mayoría de los países del mundo. Es fundamental que, al iniciar un nuevo programa, se tome una decisión sobre el alcance del cribado, definiendo la estrategia de selección de la prueba a utilizar. El objetivo del cribado de HC debe ser "detectar todas las formas de $\mathrm{HC}$ primario: leve, moderada y grave, con un esfuerzo especial para detectar aquellos pacientes HC grave, donde la morbilidad es alta si la enfermedad no se detecta y trata hasta varios meses después del nacimiento" (Grüters \& Krude, 2011). La prueba más usada para detectar HC primario es a través de la TSH, esto se debe a que la deficiencia 
de yodo, es una de las causas prevenibles más comunes de retraso mental, discapacidades del desarrollo y $\mathrm{HC}$ en todo el mundo, "la PCN para $\mathrm{HC}$ con TSH también es una herramienta excelente para monitorear el estado nutricional de yodo en las poblaciones neonatal y materna". (Zimmermann, Aeberli, Torresani, \& Burgi, 2005)

\section{Protocolos de detección}

El cribado de $\mathrm{HC}$ es eficaz para el análisis de sangre del cordón umbilical, así como para la sangre recogida en papel de filtro después de las $24 \mathrm{~h}$ de edad.

El momento ideal para la recogida de muestras es de 48 a 72 h de edad. La sangre se aplica directamente al papel de filtro y la tarjeta se envía inmediatamente después de secarse al laboratorio para su análisis. El método TSH detecta el HC primario con mayor eficacia que el cribado con T4. El análisis de T4 seguido de una prueba confirmatoria de TSH tiene el inconveniente de que faltan casos de formas leves de HC primario. Por otro lado, si la estrategia del programa de cribado lo define, este enfoque puede detectar casos de $\mathrm{HC}$ central $(\mathrm{CCH})$. Para ser efectivos, los protocolos para la detección de $\mathrm{CCH}$ se basan en uno de dos enfoques: una determinación simultánea de T4 y TSH en DBS o una combinación de T4 seguida de una prueba secundaria de TSH y, en un tercer paso de T4-glóbulo de unión (TBG). La inclusión de TBG determinaciones disminuye el número de resultados falsos positivos. (Flores \& Coronado, 2018)

Debido a que el cribado de HC suele estar formado en programas integrales de PCN, se debe hacer algún compromiso con respecto al momento de la recolección de muestras. Los partos domiciliarios, el alta temprana y las crecientes presiones económicas sobre los sistemas de atención de la salud en todo el mundo han hecho que el momento de la recogida de muestras se adelante hasta menos de $24 \mathrm{~h}$ después del nacimiento. Por tanto, es importante que en el laboratorio de selección los resultados de la prueba de cribado de TSH se interpreten en relación con el momento del muestreo, es decir, utilizando valores de corte estratificados.

Además, los resultados del cribado de HC obtenidos en algunos grupos de recién nacidos con riesgo de HC deben evaluarse por separado. Es habitual que en los neonatos prematuros (edad gestacional menor de 37 semanas), aquellos con peso al nacer menor de $2.000 \mathrm{gr}$ y todos los neonatos ingresados en una unidad de cuidados intensivos "se les tomen más de 1 muestra para la prueba; una dentro de las primeras $48 \mathrm{~h}$ y otra alrededor de los 14 días de edad o al momento del alta". (Rellan \& Garcia, 2008)

De manera similar, en el caso de que se recolecte una muestra en las primeras $24 \mathrm{~h}$ en el caso de partos múltiples (particularmente de gemelos del mismo sexo), debe haber al menos una segunda muestra recolectada. En todos estos casos, la interpretación de los valores de TSH debe realizarse teniendo en cuenta todas las muestras tomadas del mismo neonato.

Varias publicaciones se han ocupado de este tema los autores Rodríguez, María, \& Guindulain, (2019) exponen que "la idea subyacente es que en estos bebés la TSH podría ser normalmente baja incluso en un caso de HC. La supresión de TSH después de la administración de fármacos en la unidad de cuidados intensivos, la inmadurez hipotalámica o la mezcla de sangre fetal en partos múltiples se citan como las causas más probables".

Por lo general, la estrategia de un cribado de HC tiene como objetivo detectar todas las formas graves de HC lo antes posible. Para este propósito, el cribado de TSH es la prueba más certera para detectar $\mathrm{HC}$ primario. Este enfoque se utiliza hoy en día en la mayoría de los programas de detección de $\mathrm{HC}$ con buenos resultados. De acuerdo con Rodríguez, María, \& Guindulain, (2019) la tasa de detección de casos de HC, tanto permanentes como aleatorios, "es de alrededor de 1: 2500 a 1: 3500 en el momento del cribado. Esto corresponde a los casos que necesitan al menos una terapia de reemplazo temporal, y no representa la incidencia "real" de HC".

En los últimos años se ha revisado el alcance y, las estrategias de varios programas de cribado de $\mathrm{HC}$, y su objetivo no es solo la detección de las formas graves de $\mathrm{HC}$, sino que cada vez más se centra en la detección de posiblemente todas las formas de HC existentes. "Los niveles de corte de detección se han reducido gradualmente y, como consecuencia se ha informado un aumento en la incidencia de detección de casos de HC". (María Fernanda, 2015)

Actualmente se están realizando estudios sobre el resultado a largo plazo para tratar de determinar si existe un riesgo de discapacidad permanente en estos casos más leves con solo una elevación moderada de TSH y niveles de T4 generalmente normales y si estos individuos presentan una tiroides permanente o disfunción. Otro cambio en el alcance del cribado de $\mathrm{HC}$ tiene como objetivo un posible uso de la prueba neonatal para detectar casos de $\mathrm{CHC}$. El fundamento de estas iniciativas es que "en algunas poblaciones la $\mathrm{CHC}$ es una enfermedad relativamente frecuente con una prevalencia similar a la de la fenilcetonuria y que está documentada la posibilidad de un resultado desfavorable en los casos de diagnóstico tardío" (Yudexis, Carmen, \& Luisa, 2016). En el lado negativo, la falta de yodo de T4 libre basado en DBS, el analito ideal para el diagnóstico de $\mathrm{CHC}$, representa un obstáculo severo. 


\section{Confirmación de hipotiroidismo congénito}

Es necesaria la confirmación de un resultado de detección positivo antes de que un bebé sea declarado un caso de HC. El cribado no es una prueba de diagnóstico y no se debe descartar la posibilidad de etiquetar incorrectamente las muestras en el vivero. En particular, en los nacimientos múltiples, el etiquetado incorrecto de las muestras ocurre con relativa frecuencia. "La confirmación del resultado del cribado patológico debe realizarse rápidamente midiendo al menos TSH y T4 libre en una nueva muestra de sangre venosa" (Medline Plus, 2020, pág. 15).

Los autores expresan Flores \& Coronado, (2018) "la terapia de reemplazo puede iniciarse inmediatamente después de extraer la muestra de sangre y sin esperar el resultado analítico". En muchos programas, también se extrae una muestra de la madre del recién nacido y se analiza en busca de hormonas tiroideas y anticuerpos tiroideos. Los anticuerpos positivos en la madre pueden ser la causa de una forma de $\mathrm{HC}$ en el bebé.

\section{Seguimiento}

El resultado del desarrollo neurológico en los niños con $\mathrm{HC}$ detectado por el PCN y que comienzan con un tratamiento temprano con hormona tiroidea es normal o casi normal. Es importante destacar que "la terapia inicial temprana con dosis altas debe constituir el primer componente de una terapia de sustitución adecuada" (María Fernanda, 2015). El seguimiento regular de la terapia para mantener los niveles adecuados de hormona tiroidea sigue siendo de suma importancia para lograr mantener la estabilización de la hormona en los bebes que presentan este tipo de condición.

\section{Conclusión}

Como se pudo observar la prueba de cribado para HC representa una gran historia de éxito y es muy probable que continúe siéndolo y expandiéndose a nivel mundial. Actualmente es de mucha envergadura la aceptación que se definen en la realización de estas pruebas a las primeras horas de nacido.

Sin embargo, a los programas de PCN cada día se le agregan nuevos trastornos a investigar, donde los resultados obtenidos cuando una gran población al nacer se somete a un PCN integral para $\mathrm{HC}$ muestra algunas formas nuevas y previamente desconocidas de disfunción tiroidea. Durante el desarrollo del proceso investigativo también se observó, que la HC es un grupo heterogéneo de trastornos con un espectro que va desde el hipotiroidismo permanente severo hasta el hipotiroidismo leve.

La importancia de la disfunción tiroidea caracterizada por una elevación tardía de la TSH sérica en lactantes prematuros y lactantes a término con enfermedades agudas requiere una evaluación adicional. Incluso 40 años después del inicio de prueba de cribado para $\mathrm{HC}$ se puede concluir que muchas de las preguntas concernientes a la etiología permanecen sin explicación.

\section{Bibliografía}

Casarez, V. (19 de Ago de 2013). Tamiz Neonatal, Hipotiroidismo congénito, Hiperplasia suprarrenal. Recuperado el 21 de Sep de 2020, de https:// es.slideshare.net/anahycrz/tamiz-neonatal-hipotiroidismo-congnito-hiperplasia-suprarrenal

Dussault, J., Coulombe, P., \& Laberge, C. (1975). Preliminary report on a mass screening program for neonatal hypothyroidism. 86: 670-674. Quebec: Pediatr .

Flores, R., \& Coronado, Z. (2018). Tamizaje neonatal de hipotiroidismo congénito, análisis de la evidencia actual y propuesta de tamizaje para la población mexicana. Science Direct, 43-52.

Grüters, A., \& Krude, H. (2011). Detection and treatment of congenital hypothyroidism. . Nat Rev Endocrinol, 8: 104-113.

María Fernanda, C. P. (2015). Hipotiroidismo congénito. Boletín Médico del Hospital Infantil de México, 140-148.

Marrero, N., \& Frometa, A. (2000). Medición de TSH, T4 y Phe en muestras de sangre del cordón umbilical en papel de filtro: impacto en el tamizaje neonatal . Biomedica, 34-38.

Medline Plus. (25 de Mar de 2020). Medline Plus. Recuperado el 21 de Sep de 2020, de https:// medlineplus.gov/spanish/pruebas-de-laboratorio/ prueba-de-tsh/

Rellan, R., \& Garcia, R. (2008). El recién nacido prematuro. Asociación Española de Pediatría, 45-67.

Rodríguez, A., María, S., \& Guindulain, C. (2019). Diagnóstico y seguimiento de los pacientes con hipotiroidismo congénito diagnosticados por cribado neonatal. Madrid: Science Direct Asociacion Española de Pediatria.

Rodríguez, A., Sanz, M., \& Dulín, E. (2017). Cribado neonatal en enfermedades endocrinológicas. Revista Especial Endocrinologia Pediatrica, 34.

Yudexis, R., Carmen, M., \& Luisa, C. (2016). DETECCIÓN TEMPRANA DE HIPOTIROIDISMO CONGÉNITO Y FENILCETONURIA A TRAVÉS DEL CRIBADO NEONATAL EN EL ESTADO COJEDES. Scielo.

Zimmermann, M., Aeberli, I., Torresani, T., \& Burgi, H. (2005). Increasing the iodine concentration in the

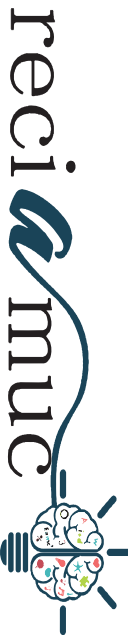


Swiss io-dized salt program markedly improved iodine status in pregnant women and children: a 5 -year prospec-tive national study. Am J Clin Nutr, 82: 388-392.

\section{CITAR ESTE ARTICULO:}

\section{(c) $(1) \odot \odot$ \\ BY NC SA}

RECONOCIMIENTO-NOCOMERCIAL-COMPARTIRIGUAL CC BY-NC-SA

ESTA LICENCIA PERMITE A OTROS ENTREMEZCLAR, AJUSTAR Y CONSTRUIR A PARTIR DE SU OBRA CON FINES NO COMERCIALES, SIEMPRE Y CUANDO LE RECONOZCAN LA AUTORÍA Y SUS NUEVAS CREACIONES ESTÉN BAJO UNA LICENCIA CON LOS MISMOS TÉRMINOS.

Calle Rueda, D., Muñoz Cueva, T., Delgado Núñez, C., \& Vera Enríquez, R. (2020). Tamizaje neonatal de hipotiroidismo congénito. RECIAMUC, 4(3), 268274. doi:10.26820/reciamuc/4.(3).julio.2020.268-274 